\title{
Multiphase transport model for heavy ion collisions at RHIC *
}

\author{
Zi-wei Lin ${ }^{\mathrm{a}}$, Subrata Pal ${ }^{\mathrm{a}}$, C.M. Ko ${ }^{\mathrm{a}}$, Bao-An Li ${ }^{\mathrm{b}}$ and Bin Zhang ${ }^{\mathrm{b}}$ \\ a Cyclotron Institute and Physics Department, Texas A\&M University, \\ College Station, Texas 77843-3366 \\ b Department of Chemistry and Physics, Arkansas State University, \\ P.O. Box 419, State University, Arkansas 72467-0419
}

Using a multiphase transport model (AMPT) with both partonic and hadronic interactions, we study the multiplicity and transverse momentum distributions of charged particles such as pions, kaons and protons in central $\mathrm{Au}+\mathrm{Au}$ collisions at RHIC energies. Effects due to nuclear shadowing and jet quenching on these observables are also studied. We further show preliminary results on the production of multistrange baryons from the strangeness-exchange reactions during the hadronic stage of heavy ion collisions.

To study heavy ion collisions at the Relativistic Heavy Ion Collider, in which a quarkgluon plasma is expected to be formed, we have developed a multiphase transport model that includes both the dynamics of initial partonic and final hadronic matters [1]. In this model, the initial space-time information for partons and strings is obtained from the HIJING model [2]. Scatterings among these minijet partons are then treated using Zhang's Parton Cascade (ZPC) [3]. After partons stop interacting, they combine with their parent strings to form hadrons using the Lund string fragmentation model [4] after an average proper formation time of $0.7 \mathrm{fm} / \mathrm{c}$. The dynamics of the resulting hadronic matter is then described by a relativistic transport model (ART) [5].

We first used the model to study heavy ion collisions at SPS energies and found that it gave smaller numbers of net baryons and kaons at midrapidity than those observed in experiments. To increase the net baryon at midrapidity, we introduced both the popcorn mechanism of baryon-antibaryon production in the Lund string fragmentation and baryonantibaryon production from and annihilation to mesons in the ART model. The kaon number was increased by adding in the ART model the production and destruction of $K^{*}$ resonances, and by adjusting the two parameters in the splitting function used in the Lund string fragmentation.

Results from the improved AMPT model for central $\mathrm{Pb}+\mathrm{Pb}$ collisions at $158 \mathrm{~A} \mathrm{GeV}$ from the SPS, corresponding to impact parameters of $b \leq 3 \mathrm{fm}$, are shown in Fig. 1 for the rapidity distributions and in Fig. $⿴$ f for the transverse momentum spectra of charged

\footnotetext{
*Supported by NSF Grants PHY-9870038 and PHY-0088934, Welch Foundation Grant A-1358, Texas Advanced Research Program Grant FY-99-010366-0081, and Arkansas Science and Technology Grant 00-B-14.
} 
particles including pions, kaons, protons, and antiprotons. We see that the theoretical results shown by solid curves agree reasonably with the experimental data [6]. On the other hand, the HIJING model with default parameters, shown by dashed curves in Fig. 2, underpredicts the inverse slopes of the transverse momentum spectra for kaons and protons in these collisions. Final state hadronic scatterings are thus important in describing the transverse momentum spectra.
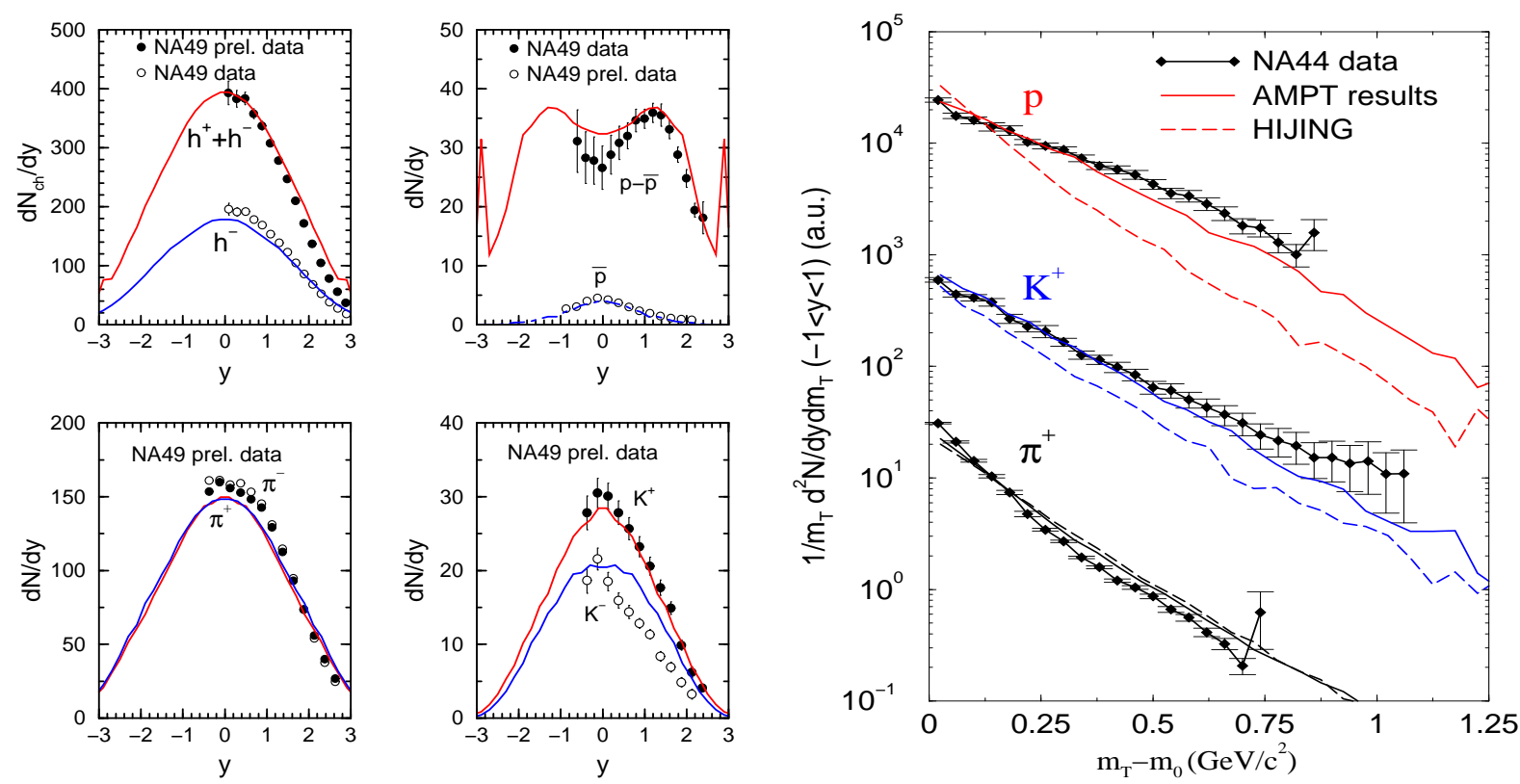

Figure 1. Rapidity distributions at SPS.

Figure 2. Transverse momentum spectra at SPS.

With parameters in the AMPT model constrained by experimental data from heavy ion collisions at SPS energies, we then studied heavy ion collisions at RHIC energies. We first show in Fig. 3 by the solid curve the pseudorapidity distribution of total charged particles in central $(b \leq 3 \mathrm{fm}) \mathrm{Au}+\mathrm{Au}$ collisions at $130 \mathrm{~A} \mathrm{GeV}$. The theoretical result is consistent with the data from the PHOBOS collaboration [0] shown in the figure by the full circle. We note that the total charged particle multiplicity increases appreciably without final hadronic scatterings but is hardly affected by partonic scatterings. The latter is partly due to the absence of inelastic scattering in the ZPC model. To model the effect of parton energy loss due to inelastic scatterings, we introduce the jet quenching as in default HIJING. In Fig. 3, we show by dashed curves the results from AMPT model with a jet quenching of $d E / d x=1 \mathrm{GeV} / \mathrm{fm}$. Also shown in Fig. 3 are the results obtained by neglecting nuclear shadowing on parton production in the AMPT model. In both cases, the total charged particle multiplicity is larger than that from the default AMPT model. The PHOBOS data is thus consistent with a significant nuclear shadowing effect but a rather weak jet quenching. Our predictions for the multiplicities of pions, kaons, protons and antiprotons are also shown by solid curves in Fig. 3. It is seen that the $\bar{p} / p$ ratio is significantly increased in comparison with that in central $\mathrm{Pb}+\mathrm{Pb}$ collisions at $\mathrm{SPS}$. 

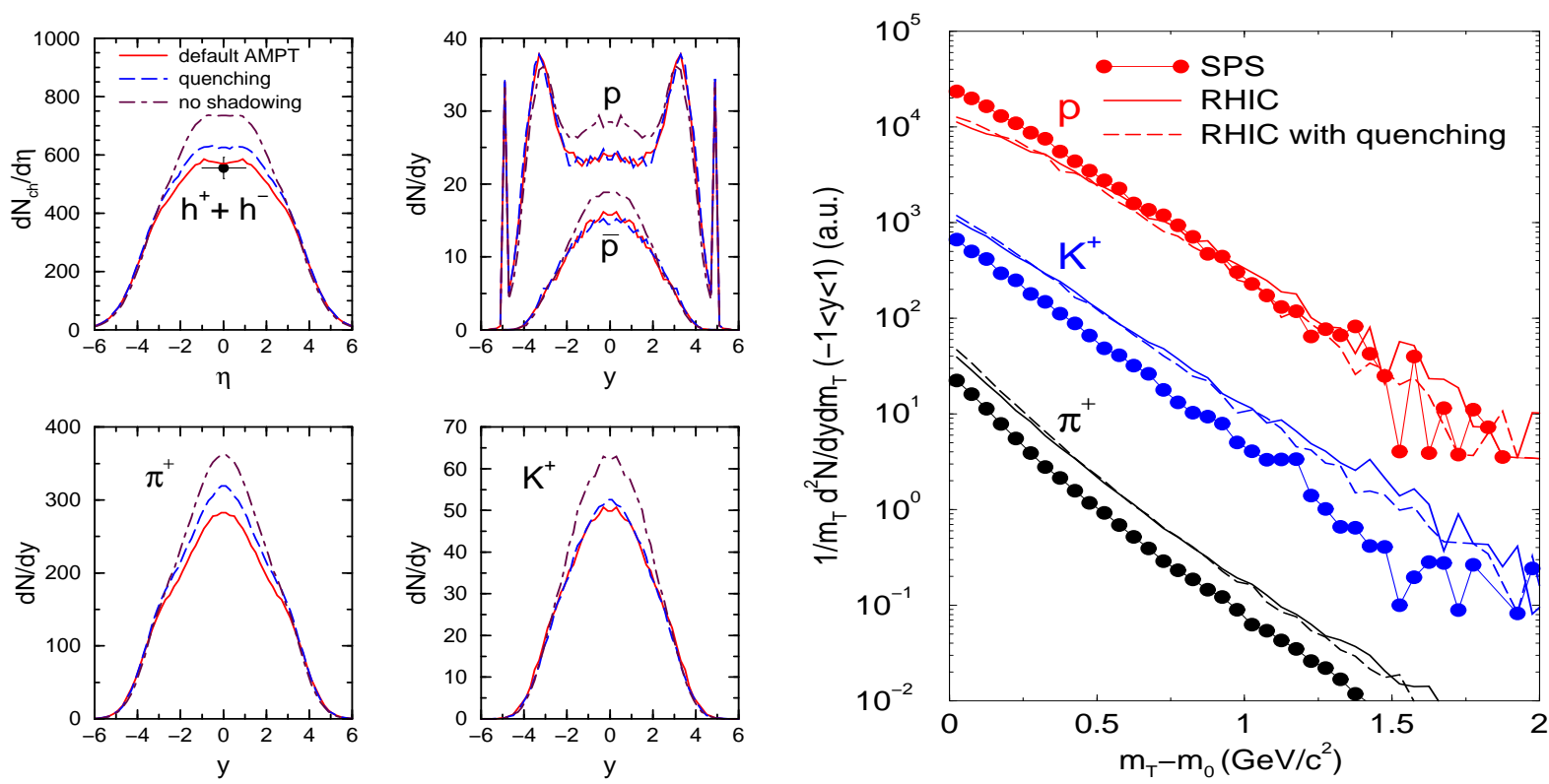

Figure 3. Rapidity distributions at RHIC.

Figure 4. Transverse momentum spectra at RHIC.

In Fig. 田, we show by solid curves the transverse momentum spectra of pions, kaons, and protons in central $\mathrm{Au}+\mathrm{Au}$ collisions at $130 \mathrm{~A} \mathrm{GeV}$. For comparison, we also show by circled curves the default AMPT results for central $\mathrm{Pb}+\mathrm{Pb}$ collisions at SPS as already shown in Fig. 2 and by dashed curves the AMPT results with a jet quenching of $d E / d x=1 \mathrm{GeV} / \mathrm{fm}$. We find that all three particles, especially protons, have larger transverse momenta at RHIC than at SPS. As expected, jet quenching suppresses moderately the yields of these particles at high transverse momenta.

We have also extended the AMPT model to include strangeness-exchange reactions in the hadronic matter in order to study the production of multistrange baryons in relativistic heavy ion collisions. This is important for understanding whether one can use enhanced production of multistrange particles as a signal for the formation of quark-gluon plasma in relativistic heavy ion collisions [8]. The cross sections for $\Lambda$ and $\Sigma$ productions from $K^{-}$ and nucleon interactions are known empirically. From the parameterized cross sections for $K^{-} p \rightarrow \pi^{0} \Lambda$ and $\pi^{0} \Sigma^{0}$, and $K^{-} n \rightarrow \pi^{-} \Lambda^{0}$ [9], the isospin-averaged cross section of hyperon production from $\bar{K} N \rightarrow \pi Y$ can be obtained, and it is $3 / 2$ times their sum. The cross sections for $\Xi$ and $\Omega$ production, i.e., $\bar{K} \Lambda \rightarrow \pi \Xi$ and $\bar{K} \Xi \rightarrow \pi \Omega$, are unknown. In our preliminary study, we assume that they are the same as that for $\bar{K} N \rightarrow \pi Y$.

Table 1

Strange particle yields at midrapidity from AMPT at SPS and RHIC $(\sqrt{s}=130 \mathrm{~A} \mathrm{GeV})$.

\begin{tabular}{llllllll}
\hline & $K^{+}$ & $K^{-}$ & $\Lambda$ & $\Xi^{-}$ & $\Omega^{-}$ & $\Xi^{-} / \Lambda$ & $\Omega^{-} / \Xi^{-}$ \\
\hline SPS & 28 & 19 & 12 & 1.1 & 0.19 & 0.093 & 0.16 \\
RHIC & 50 & 45 & 13 & 2.0 & 0.43 & 0.16 & 0.22 \\
RHIC $\left(P_{s}=P_{u}\right)$ & 68 & 61 & 13 & 5.1 & 1.9 & 0.38 & 0.37 \\
\hline
\end{tabular}


In Table 1, we show the abundance of strange hadrons at midrapidity $(|y|<0.5)$ from the AMPT model that includes the above strangeness-exchange reactions and their inverse reactions. We see that our results for central $\mathrm{Pb}+\mathrm{Pb}$ collisions at $\mathrm{SPS}(\sqrt{s}=17 \mathrm{~A} \mathrm{GeV})$ are consistent (within 50\%) with the large enhancement of multistrange particle production observed in the experiments. We note that a similar conclusion was reached in Ref. [10] using a different assumption on the cross sections of strangeness-exchange reactions. Although the AMPT model includes a partonic stage, no strange quark production is considered at present. To simulate the effect of strange particle production during the partonic stage of heavy ion collisions, we let strange quarks to have the same production probability as light quarks in the Lund string fragmentation. The results from such a scenario are shown in the last row of Table 1. It is seen that this leads to a significant enhancement of multistrange particle production, although its effect on particles with single strangeness, such as $K$ and $\Lambda$, is much smaller. Our results thus imply that enhanced production of multistrange particles in relativistic heavy ion collisions remains a possible signal for the initial quark-gluon plasma in spite of multistrange particle production from the hadronic matter.

To summarize, a multiphase transport model has been developed for heavy ion collisions at RHIC energies. Comparisons of the theoretical results with the PHOBOS data on total charged multiplicity indicate that there is a significant nuclear shadowing but a weak jet quenching in the initial stage of collisions. The predicted transverse momentum spectra of particles are found to have larger inverse slopes at RHIC than at SPS. We have also studied the effect of strangeness-exchange reactions on the production of multistrange particles and found that the observed enhancement in heavy ion collisions at SPS can be largely explained. For heavy ion collisions at RHIC, strangeness-exchange reactions also enhance the yield of multistrange particles. Allowing enhanced production of strange particles during hadronization further increases significantly the ratios of multistrange to single-strange particles. Multistrange particle production is thus a sensitive probe to the early dynamics of heavy ion collisions.

\section{REFERENCES}

1. B. Zhang, C.M. Ko, B.A. Li and Z.W. Lin, Phys. Rev. C 61 (2000) 067901; Z.W. Lin, S. Pal, C.M. Ko, B.A. Li and B. Zhang, eprint No. nucl-th/0011059.

2. X.N. Wang and M. Gyulassy, Phys. Rev. D 44 (1991) 3501.

3. B. Zhang, Comp. Phys. Comm. 109 (1998) 193.

4. T. Sjöstrand, Comp. Phys. Comm. 82 (1994) 74.

5. B.A. Li and C.M. Ko, Phys. Rev. C 52 (1995) 2037.

6. I.G. Bearden et al. (NA44 Collaboration), Phys. Rev. Lett. 78 (1997) 2080; H. Appelshäuser et al. (NA49 Collaboration), Phys. Rev. Lett. 82 (1999) 2471; F. Siklér et al. (NA49 Collaboration), Nucl. Phys. A 661 (1999) 45c; S. Jeon and J. Kapusta, Phys. Rev. C 63 (2001) 011901.

7. B.B. Back et al., PHOBOS Collaboration, Phys. Rev. Lett. 85 (2000) 3100.

8. J. Rafelski and B. Müller, Phys. Rev. Lett. 48 (1982) 1066; 56 (1986) 2334 (E).

9. J. Cugnon, P. Deneye and J. Vandermeulen, Phys. Rev. C 41 (1990) 1701.

10. S. Vance, J. Phys. G 27 (2001) 603. 